\title{
M-COMMERCE: UM NOVO CONHECIMENTO ISOLADO?
}

\section{M-COMMERCE: A NEW ISOLATED KNOWLEDGE?}

\author{
Wilnei Aldir Schneider ${ }^{1}$ \\ Micheline Gaia Hoffmann ${ }^{1}$ \\ Rafael Tezza ${ }^{1}$
}

Recebido em: 24/06/2016 Aceito em: 24/11/2016

was.was@hotmail.com

Resumo: O objetivo desta pesquisa foi analisar se a literatura de inovação é aplicável aos estudos sobre m-commerce que analisam os ofertantes do serviço. Realizou-se uma busca sistemática na literatura, sobre $m$-commerce, em nove bases de dados científicas. Os artigos encontrados foram analisados qualitativamente e os dados obtidos foram analisados à luz da literatura de inovação empresarial. Os resultados encontrados mostram que esta literatura é compatível com o que tem sido encontrado nas pesquisas sobre $m$-commerce, mas não tem sido utilizada. Este trabalho buscou contribuir para o preenchimento desta lacuna do conhecimento. Além da contribuição teórica, contribuições práticas também são mencionadas.

Palavras-chave: M-commerce. Inovação. Serviço. Gestão.

Abstract: This research aimed to analyze if the literature of innovation is applicable to the studies on m-commerce that analyze the offerors of the service. It was carried out a systematic literature search about m-commerce in nine scientific databases. The found articles were analyzed qualitatively and the data were analyzed according to business innovation literature. The results show that this literature is compatible with the results found in research on $\mathrm{m}$ commerce, but have not been used. This study sought to contribute to filling this knowledge gap. Besides the theoretical contributions, practical contributions are also mentioned.

Keywords: M-commerce. Innovation. Service. Management.

\footnotetext{
${ }^{1}$ Universidade do Estado de Santa Catarina - UDESC - Florianópolis - Santa Catarina - Brasil. Agradecemos à Fundação de Amparo à Pesquisa e Inovação do Estado de Santa Catarina (FAPESC) pelo auxílio financeiro recebido em forma de Bolsa de Estudos durante a realização desta pesquisa
} 


\section{INTRODUÇÃO}

A partir de 1990, o comércio, da forma como era conhecido até então, passou por várias mudanças com a chegada do comércio eletrônico (e-commerce), que trouxe uma nova forma de conduzir os negócios (NGAl; GUNASEKARAN, 2005). Com o tempo, novas tecnologias foram surgindo, dentre elas, os dispositivos móveis com acesso sem fio à Internet (FROLICK; CHEN, 2004). Essas tecnologias, além de marcar uma era de comunicação em massa, também têm marcado uma era de comércio para as massas, pois o que antes exigia um ponto fixo de conexão à Internet, com os dispositivos móveis (celulares, smartphones, tablets e outros que permitem o acesso móvel à Internet) pode ser feito a partir de qualquer lugar (BHEDA, 2010).

Com isso, surgiu uma nova forma de comércio, o m-commerce, que é uma forma específica de comércio eletrônico realizado por meio de dispositivos móveis que tenham conexão com a Internet (MAY, 2001; JONKER, 2003; CHONG, 2013; IVAN; MILODIN; ZAMFIROIU, 2013). Esse serviço tornou-se possível a partir do desenvolvimento tecnológico da telefonia móvel (JIN; VILLEGAS, 2008), que fez surgir uma ferramenta de marketing com grandes possibilidades como canal de compras (MARTíN-GUTIÉRREZ; LÓPEZ-CATALÁN; RAMÓN-JERÓNIMO, 2012). Consequentemente, também com grandes possibilidades como canal de vendas. Dentro dessa perspectiva, Huang, Qi e Dong (2007) e Godoe e Hansen (2009) trazem à discussão a necessidade de se ter um modelo de negócios adequado ao serviço em questão.

De acordo com o relatório Webshoppers, publicado pelo E-bit (2015), que traz dados sobre comércio eletrônico no Brasil, o uso do $m$-commerce, nos últimos anos, tem crescido de forma ininterrupta. Em janeiro de 2014 apenas 4,8\% das transações de comércio eletrônico eram realizadas por m-commerce. Valor que, um ano antes, era de 2,5\%. Já em janeiro de 2015, a participação do $m$ commerce no e-commerce passou para 9,7\%, considerando ainda que durante o ano de 20140 comércio eletrônico como um todo teve aumento real de 26,7\% (E-BIT, 2015). A utilização da telefonia móvel também tem apresentado crescimento ininterrupto ao longo dos anos, atingindo a faixa das 280 milhões de linhas ativas no Brasil em dezembro de 2014, número 3,55\% superior ao registrado 12 meses antes, conforme dados da Anatel (http://www.anatel.gov.br). Estes números apontam para a importância e a oportunidade das empresas em inovar com a introdução do $\mathrm{m}$ commerce como um novo canal de vendas e/ou relacionamento com os clientes.

Diferentemente da maior parte das pesquisas sobre m-commerce, que reúnem seus esforços para analisar os usuários do serviço, tal como os trabalhos de Wu e Wang (2005), Yang (2005), Chong (2013) e Malik, Kumra e Srivastava (2013), o interesse deste trabalho está voltado aos ofertantes do serviço de m-commerce, tal como os artigos de Huang, Qi e Dong (2007) e Shih e Huang (2009). Em outras palavras, às empresas que utilizam o m-commerce como canal de vendas e/ou relacionamento com seus clientes.

Mesmo que o m-commerce seja visto como uma oportunidade de inovação para as empresas (SCHIERHOLZ; KOLBE; BRENNER, 2005), constatou-se, por meio de uma revisão da literatura, na qual buscou-se trabalhos que analisam as empresas ofertantes do serviço, que nenhuma das pesquisas realizadas utiliza a literatura de inovação empresarial como base teórica do estudo. Nesse 
contexto surgiu a questão que motivou o presente estudo: Será que a literatura de inovação é aplicável à forma como o m-commerce tem sido visto, ou entendido, pelos pesquisadores?

Para responder a esta questão, este estudo realiza uma revisão teórica sobre a teoria clássica da inovação, abordando importantes autores da área. Parte-se, principalmente, dos entendimentos sobre o termo Inovação adotados pela Organização para a Cooperação e Desenvolvimento Econômico [OCDE] (2005) e por Christensen (2012) e das classificações de Sundbo e Gallouj (1998), Davila, Epstein e Shelton (2007) e Tidd, Bessant e Pavitt (2008). À luz do entendimento teórico destes autores, analisam-se os estudos de $m$-commerce. O objetivo do artigo é analisar se a literatura de inovação é aplicável aos estudos sobre $m$-commerce que analisam os ofertantes do serviço.

A estrutura deste artigo resume-se, além do tópico introdutório, aos tópicos de inovação, método, apresentação dos dados, análise e considerações finais.

\section{INOVAÇÃO}

Durante muito tempo, as organizações conseguiam desfrutar de alguma vantagem competitiva apenas se tivessem um tamanho ou um patrimônio que Ihes proporcionasse essa vantagem. Com o advento das inovações, o cenário vem alterando-se, proporcionando vantagem competitiva não às organizações com maior tamanho ou recursos, mas às organizações que conseguem articular conhecimento para conceber, criar e lançar novos produtos ou serviços (TIDD; BESSANT; PAVITT, 2008).

O termo Inovação, a partir das definições da OCDE (2005), pode ser entendido como a implementação de algo (produto/serviço, processo, método organizacional ou método de marketing) novo ou significativamente melhorado. Neste escopo, a literatura traz algumas classificações, sendo parte delas apresentadas no Quadro 1. 
Quadro 1 - Classificações da literatura de inovação empresarial

\begin{tabular}{|c|c|c|c|c|c|c|c|}
\hline \multicolumn{8}{|c|}{ FOCO DA INOVAÇÃO } \\
\hline & Produto & \multicolumn{2}{|c|}{ Processo } & \multicolumn{2}{|c|}{ Organizacional } & $\begin{array}{l}\text { Mercado ou } \\
\text { marketing }\end{array}$ & Ad hoc \\
\hline $\begin{array}{c}\text { O que } \\
\text { é? }\end{array}$ & $\begin{array}{l}\text { Introdução } \\
\text { de um } \\
\text { produto ou } \\
\text { serviço novo } \\
\text { ou significa- } \\
\text { tivamente } \\
\text { aprimorado. }\end{array}$ & \multicolumn{2}{|c|}{$\begin{array}{l}\text { Renovação } \\
\text { ou aprimora- } \\
\text { mento das } \\
\text { instruções de } \\
\text { produção e } \\
\text { entrega do } \\
\text { produto ou } \\
\text { serviço. }\end{array}$} & & $\begin{array}{l}\text { nentação } \\
\text { na nova } \\
\text { ma de } \\
\text { ciamento } \\
\text { egócios. }\end{array}$ & $\begin{array}{c}\text { Consiste em um } \\
\text { novo } \\
\text { posicionamento } \\
\text { de mercado, } \\
\text { introduzindo } \\
\text { novos métodos } \\
\text { de marketing, } \\
\text { explorando novos } \\
\text { nichos de } \\
\text { mercado, bem } \\
\text { como novos } \\
\text { segmentos. }\end{array}$ & $\begin{array}{c}\text { Processo } \\
\text { conduzido em } \\
\text { parceria entre } \\
\text { fornecedor e } \\
\text { cliente e não } \\
\text { pode ser } \\
\text { replicado, no } \\
\text { entanto, o } \\
\text { fornecedor } \\
\text { permanece com } \\
\text { a experiência e } \\
\text { competências } \\
\text { adquiridas. É } \\
\text { específica para } \\
\text { inovação em } \\
\text { serviços cuja } \\
\text { base é o } \\
\text { conhecimento. }\end{array}$ \\
\hline $\begin{array}{l}\text { Refe- } \\
\text { rência }\end{array}$ & \multicolumn{6}{|c|}{ Sundbo e Gallouj (1998); OCDE (2005) } & $\begin{array}{c}\text { Sundbo e Gallouj } \\
\text { (1998) }\end{array}$ \\
\hline \multicolumn{8}{|c|}{ GRAUS DE INOVAÇÃO } \\
\hline & \multicolumn{2}{|c|}{ Incremental } & \multicolumn{2}{|c|}{ Radical } & \multicolumn{3}{|c|}{ De Ruptura } \\
\hline $\begin{array}{c}\text { O que } \\
\text { é? }\end{array}$ & \multicolumn{2}{|c|}{$\begin{array}{l}\text { Melhoria de } \\
\text { produtos já } \\
\text { estabelecidos, } \\
\text { visando atingir } \\
\text { melhor } \\
\text { desempenho } \\
\text { sem, no entanto, } \\
\text { desconsiderar as } \\
\text { dimensões de } \\
\text { desempenho que } \\
\text { os clientes têm } \\
\text { historicamente } \\
\text { valorizado. }\end{array}$} & \multicolumn{2}{|c|}{$\begin{array}{l}\text { Criação de } \\
\text { produtos } \\
\text { totalmente } \\
\text { novos, cujas } \\
\text { características } \\
\text { ou uso } \\
\text { pretendido } \\
\text { diferem } \\
\text { totalmente de } \\
\text { qualquer } \\
\text { produto } \\
\text { produzido } \\
\text { anteriormente. }\end{array}$} & \multicolumn{3}{|c|}{$\begin{array}{l}\text { Inovação que leva, ao menos no curto prazo, a } \\
\text { pior desempenho dos produtos, porém é o tipo de } \\
\text { inovação que mais tem levado empresas líderes } \\
\text { ao fracasso. Inovações de ruptura trazem } \\
\text { proposições de valor bastante diferentes daquelas } \\
\text { que existiam anteriormente. Mesmo que o } \\
\text { desempenho desses produtos seja inferior aos } \\
\text { estabelecidos, eles têm aceitação pelo mercado } \\
\text { por apresentarem outras características, que } \\
\text { proporcionam valor ao cliente. Dentre as } \\
\text { vantagens, é que esses produtos normalmente } \\
\text { são "mais baratos, mais simples, menores e } \\
\text { frequentemente mais convenientes de usar". }\end{array}$} \\
\hline $\begin{array}{l}\text { Refe- } \\
\text { rência }\end{array}$ & \multicolumn{4}{|c|}{ OCDE (2005) } & \multicolumn{3}{|c|}{ Christensen (2012, p. 24) } \\
\hline \multicolumn{8}{|c|}{ ATIVIDADES INOVATIVAS } \\
\hline & \multicolumn{2}{|c|}{ Tecnológica } & \multicolumn{5}{|c|}{ Estratégica } \\
\hline $\begin{array}{c}\text { O que } \\
\text { é? }\end{array}$ & \multicolumn{2}{|c|}{$\begin{array}{c}\text { Pesquisa e } \\
\text { desenvolvimento } \\
\text { de novos } \\
\text { produtos/ } \\
\text { serviços. }\end{array}$} & \multicolumn{5}{|c|}{$\begin{array}{l}\text { Definição do modelo de negócio, que consiste na forma como a } \\
\text { empresa cria, vende e proporciona valor aos seus clientes. Há três } \\
\text { áreas em que o modelo de negócios pode orientar a inovação: } \\
\text { proposição de valor - refere-se ao que é vendido e lançado no } \\
\text { mercado, podendo ser um produto ou serviço com inovações radicais } \\
\text { ou incrementais; cadeia de suprimentos - consiste na forma como o } \\
\text { valor é criado e entregue ao mercado; e cliente-alvo - consiste na } \\
\text { exploração de um diferente nicho de mercado. }\end{array}$} \\
\hline $\begin{array}{l}\text { Refe- } \\
\text { rência }\end{array}$ & \multicolumn{7}{|c|}{ Davila, Epstein e Shelton (2007) } \\
\hline
\end{tabular}

Fonte: Elaborado pelos autores (2015).

Independentemente do foco da inovação, sua gestão requer mudanças na percepção estratégica da organização (HIDALGO; ALBORS, 2008). A inovação é um elemento que deve ser 
considerado parte integrada na mentalidade de um negócio, devendo estar alinhada à estratégia organizacional (DAVILA; EPSTEIN; SHELTON, 2007).

Em relação aos graus de inovação (Quadro 1), mais especificamente inovação de ruptura, Christensen (2012) apresenta cinco princípios: a) as companhias dependem dos clientes e dos investidores para obter recursos; b) mercados pequenos não solucionam a necessidade de crescimento de grandes empresas; c) mercados que não existem não podem ser avaliados; d) as capacidades de uma organização definem suas incapacidades - diferentes contextos requerem diferentes capacidades organizacionais; e) o fornecimento de tecnologia pode não ser igual à demanda do mercado - o mercado pode não estar preparado para absorver esses produtos e cria-se um vácuo nos pontos de preço inferiores, o que dá abertura para a entrada de competidores que empregam tecnologias de ruptura.

Já quanto às atividades inovativas (Quadro 1), Hidalgo e Albors (2008) afirmam que obstáculos podem impor-se ao seu gerenciamento. Para os autores, o principal obstáculo consiste em acreditar que inovação requer esforço extra, tempo, motivação e dinheiro. Além disso, algumas técnicas de gerenciamento de inovação são consideradas muito mais acadêmicas do que práticas. Outra dificuldade é a de que muitas empresas não têm capacidade de identificar inovações e incorporá-las ao processo normal de produção. Em uma linha semelhante, Tidd, Bessant e Pavitt (2008) comentam que há fatores do contexto que podem exercer influências importantes no processo de inovação. Esses fatores podem ser agrupados em: contexto estratégico da inovação, capacidade inovadora da organização, e conexão entre a organização e os elementos essenciais do cenário externo. Davila, Epstein e Shelton (2007) ainda apontam para a importância das colaborações, tanto interna quanto externa, afirmando que ambas são indispensáveis para o processo de inovação.

As colaborações externas, especificamente, dependem de alguns fatores que contribuem para o sucesso da atividade inovativa: a aliança é percebida como importante por todos os parceiros; existe um líder na colaboração; existe considerável grau de confiança entre os parceiros; estabelecem-se um claro planejamento de projeto e tarefas-marco definidas; existe frequente comunicação entre os parceiros, particularmente entre o pessoal de marketing e o pessoal técnico; as partes colaboradoras contribuem como esperado; e os benefícios são percebidos como igualmente distribuídos (TIDD; BESSANT; PAVITT, 2008).

Retornando às dificuldades, a complexidade burocrática, a baixa consciência da inovação entre os gestores e a incapacidade de compartilhar conhecimento também são considerados obstáculos que impõem-se ao gerenciamento da inovação. Além destes, ainda existem outros, que são: necessidade de investimento financeiro, dificuldade de aceitar falhas, burocracia excessiva e incerteza, e falta de programas de formação e superação de complicações culturais (HIDALGO; ALBORS, 2008).

Enfim, o que influencia a decisão de uma empresa de querer inovar ou não? E o que leva uma empresa a cooperar ou buscar cooperação? O Quadro 2 apresenta duas dimensões, e respectivos desdobramentos, que afetam a atitude de uma empresa em relação à inovação. 
Quadro 2 - Dimensões que afetam a atitude em relação à inovação

\begin{tabular}{|c|c|l|}
\hline Dimensões & Variáveis & \multicolumn{1}{c|}{ O que significa? } \\
\hline \multirow{4}{*}{$\begin{array}{c}\text { Características } \\
\text { da tecnologia } \\
\text { (inovação) }\end{array}$} & $\begin{array}{c}\text { Complexidade da } \\
\text { inovação }\end{array}$ & $\begin{array}{l}\text { Devido à interdisciplinaridade de diversas tecnologias, tem- } \\
\text { ino buscado alavancar as competências internas utilizando } \\
\text { tecnologias disponíveis externamente. }\end{array}$ \\
\cline { 2 - 4 } & $\begin{array}{c}\text { Capacidade de } \\
\text { codificação }\end{array}$ & $\begin{array}{l}\text { Consiste no quanto um conhecimento sobre dada } \\
\text { tecnologia é passível de ser descrito para possibilitar sua } \\
\text { transferência. }\end{array}$ \\
\cline { 2 - 4 } & $\begin{array}{c}\text { Potencial de } \\
\text { credibilidade }\end{array}$ & $\begin{array}{l}\text { Credibilidade dada a uma empresa por determinada } \\
\text { tecnologia. }\end{array}$ \\
\hline \multirow{4}{*}{$\begin{array}{c}\text { Estratégia } \\
\text { emprança da }\end{array}$} & $\begin{array}{l}\text { Grau em que a estratégia empresarial determina uma } \\
\text { política de diferenciação tecnológica ou de liderança. }\end{array}$ \\
\cline { 2 - 4 } & $\begin{array}{c}\text { Capacidades e } \\
\text { conhecimento } \\
\text { técnico }\end{array}$ & $\begin{array}{l}\text { Capacidades técnicas que uma empresa possui } \\
\text { internamente. }\end{array}$ \\
\cline { 2 - 4 } & $\begin{array}{c}\text { Cultura da } \\
\text { empresa }\end{array}$ & Maneira como cada empresa desenvolve suas atividades. \\
\cline { 2 - 4 } & $\begin{array}{c}\text { Conforto da } \\
\text { administração } \\
\text { com determinada } \\
\text { área técnica }\end{array}$ & $\begin{array}{l}\text { Variável que pode assumir uma linha de familiaridade com } \\
\text { determinada tecnologia, ou uma linha de confiança na } \\
\text { capacidade da equipe em ser bem sucedida com uma nova } \\
\text { área técnica. }\end{array}$ \\
\hline
\end{tabular}

Fonte: Elaborado pelos autores (2015), com base em Tidd, Bessant e Pavitt (2008).

Nybakk e Jenssen (2012) conduzem um estudo no qual avaliam estratégia de inovação, desempenho financeiro e ambiente de trabalho inovador. Os resultados atingidos apontam que a estratégia de inovação e um ambiente de trabalho inovador estão positivamente relacionados ao desempenho financeiro. Os autores afirmam que os resultados corroboram resultados de estudos anteriores.

De posse das classificações da literatura, das barreiras que aparecem na gestão, de motivos que levam as empresas a cooperar ou buscar cooperação, e também de alguns benefícios proporcionados pela inovação, o próximo tópico apresenta o método de pesquisa empregado para desenvolvimento do estudo.

\section{MÉTODO}

Este trabalho trata-se de uma pesquisa documental, qualitativa, realizada com dados secundários. Os dados foram obtidos a partir de uma busca sistemática na literatura, na qual foram buscados artigos que discutem o m-commerce na condição do ofertante do serviço. A busca foi realizada com base em uma adaptação do modelo proposto por Villas, Macedo-Soares e Russo (2008), conforme Figura 1. 
Figura 1 - Método de seleção de documentos

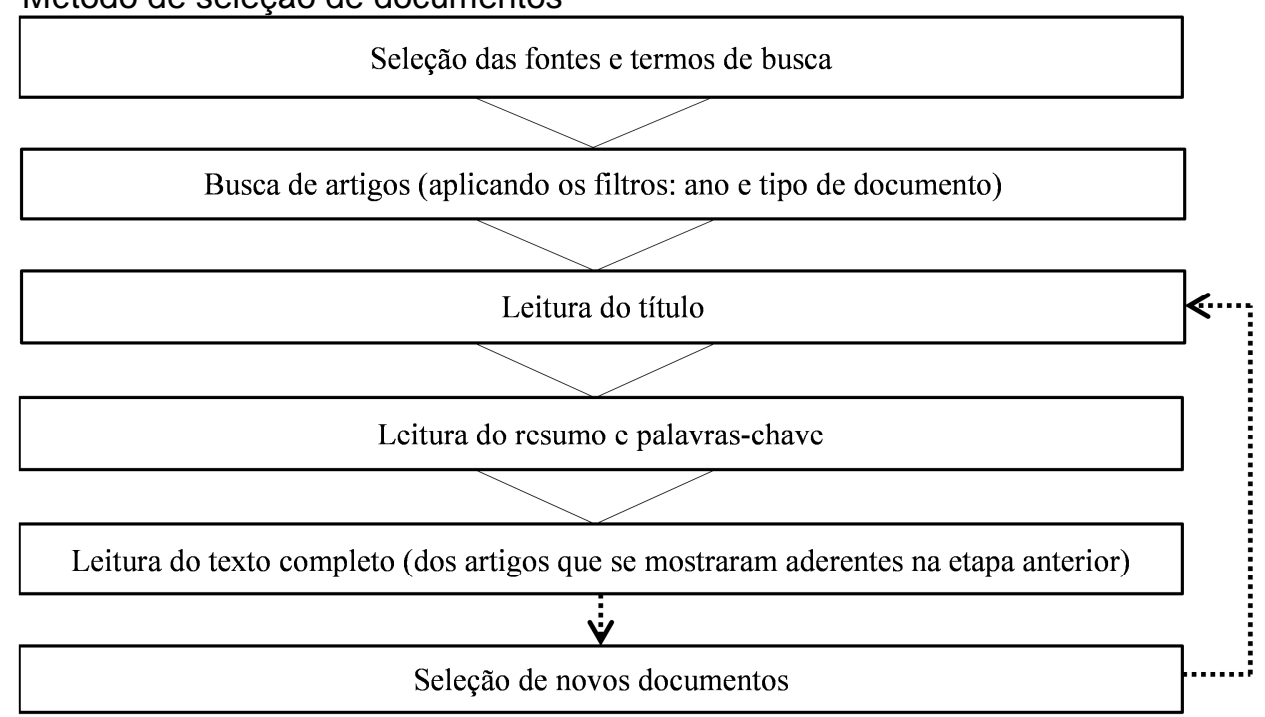

Fonte: Elaborado pelos autores (2015), com base em Villas, Macedo-Soares e Russo (2008).

Para a seleção das fontes e termos de busca, os autores partiram de conhecimento anterior, adquirido em revisão de literatura mais ampla sobre m-commerce. No presente estudo, fez-se necessária uma delimitação inicial mais direcionada ao objetivo da pesquisa. Dessa forma, utilizaramse como fontes as seguintes bases de dados científicas: ACM, EBSCO, Emerald, Academic OneFile (GALE), Scielo, Science Direct, Springer, Web Of Science, e Wiley. Quanto aos termos de busca, utilizaram-se os seguintes:

1. ("m-commerce" OR "mobile commerce") AND "case study";

2. ("m-commerce" OR "mobile commerce") AND ("organi?ation" AND "use");

3. ("m-commerce" OR "mobile commerce") AND "innovation";

4. ("m-commerce" OR "mobile commerce") AND "business use";

5. ("m-commerce" OR "mobile commerce") AND "seller";

6. ("m-commerce" OR "mobile commerce") AND "strategic planning";

7. ("m-commerce" OR "mobile commerce") AND "marketing";

8. ("m-commerce" OR "mobile commerce") AND "revenue".

A seleção destes termos foi realizada considerando as seguintes situações: casos sobre organizações que oferecem o serviço de $m$-commerce, uso do $m$-commerce em organizações, $m$ commerce entendido como uma forma de inovação para quem passa a oferecer o serviço, uso do $\mathrm{m}$ commerce em negócios, $m$-commerce como uma ferramenta adotada por vendedores, planejamento estratégico que já inclui o $m$-commerce como canal de vendas e/ou relacionamento, $m$-commerce como uma ferramenta de marketing e m-commerce como uma forma de aumento das receitas. Alguns destes termos foram elencados com base nas revisões de literatura de Ngai e Gunasekaran (2007) e Varnali e Toker (2010) e outros foram estabelecidos a partir de uma revisão mais ampla da literatura sobre $m$-commerce.

$\mathrm{Na}$ etapa seguinte, buscaram-se os documentos, limitando os termos de busca, apresentados anteriormente, aos campos de título, palavra-chave e resumo, quando a base de dados consultada permitia fazê-lo. Nos demais casos (bases ACM, Springer e Web Of Science), buscaram-se os termos 
de busca no texto completo. Além disso, já na busca, inseriu-se os filtros de "ano de publicação" e "tipo de documento". Com base no tema da pesquisa e na época em que o assunto começou a ganhar destaque, o período de publicação considerado neste trabalho foi de janeiro de 2000 até a data da pesquisa, agosto de 2014. A data de início do período selecionado é a mesma utilizada na revisão de literatura sobre $m$-commerce realizada por Ngai e Gunasekaran (2007). Quanto ao filtro "tipo de documento", buscou-se artigos publicados em anais de congressos (Proceedings) e artigos publicados em revistas científicas (Journals). Tanto os artigos de Journal quanto os de Proceedings foram buscados [apenas] nas bases de dados mencionadas anteriormente, as quais veiculam trabalhos nacionais e internacionais. Buscaram-se documentos escritos nos idiomas português e inglês.

A busca inicial resultou em 1336 referências não duplicadas. Foi utilizado o software EndNote $X 7^{\circledR}$ para organização do material. O passo seguinte foi a leitura dos títulos para eliminar referências não aderentes ao objetivo da pesquisa. Dos 257 artigos que restaram, leram-se as palavras-chave e o resumo e constatou-se que apenas 22 poderiam estar relacionados ao que se buscava. Entretanto, ao ler e analisar os textos completos, foram eliminados mais seis artigos, que não atendiam aos objetivos da pesquisa. Cabe destacar que somente obteve-se acesso aos artigos disponíveis em bases de dados assinadas pela CAPES.

O próximo passo foi a busca por novos documentos, seguindo o método de Chang, Cheung e Lai (2005). Para tal, selecionou-se a revista Industrial Management and Data Systems, pois é uma das revistas encontradas na busca inicial e que tem publicado vários estudos sobre $m$-commerce. Nenhum artigo novo foi encontrado. Dessa forma, coletaram-se e analisaram-se os resultados reportados nos dezesseis artigos restantes. Cabe destacar que nenhum estudo brasileiro sobre o tema foi encontrado.

Os dados para análise foram extraídos dos artigos por meio de análise descritiva do conteúdo, na qual se buscou identificar os principais resultados atingidos nas pesquisas. Após a coleta, os dados foram organizados e, em seguida, analisados à luz da literatura de inovação empresarial, buscando verificar se essa literatura é aplicável ao serviço de m-commerce.

\section{APRESENTAÇÃO DOS DADOS}

A partir da revisão de literatura e dos dados coletados, verificou-se que é possível reunir os resultados reportados nos artigos em quatro grupos: fatores de influência à oferta do serviço de $\mathrm{m}$ commerce; benefícios e efeitos obtidos com a oferta do serviço de $m$-commerce; barreiras para a oferta do serviço de m-commerce; e fatores críticos de sucesso na oferta do serviço de $m$-commerce. A nomenclatura atribuída a cada um dos grupos baseou-se em seu conteúdo.

Os dados apresentados não pretendem ser exaustivos, eles apenas representam os principais resultados dos estudos e que têm uma possível ligação com a literatura de inovação empresarial. Nem todos os trabalhos analisados reportam dados que se inserem em cada um dos quatro grupos mencionados anteriormente. No Quadro 3, apresentam-se os fatores que influenciam a 
decisão de uma empresa/organização oferecer o serviço de $m$-commerce, que é o primeiro grupo dos quatro citados anteriormente.

Quadro 3 - Fatores de influência à oferta do serviço de $m$-commerce

\begin{tabular}{|l|c|c|}
\hline O que pode levar as organizações a oferecerem o m-commerce & $\begin{array}{c}\text { Tipo de } \\
\text { estudo }\end{array}$ & Referência \\
\hline $\begin{array}{l}\text { Busca por melhorar o relacionamento com os consumidores e } \\
\text { aumentar as receitas. }\end{array}$ & $\begin{array}{c}\text { Estudo de } \\
\text { caso }\end{array}$ & $\begin{array}{c}\text { Huang, Qi e } \\
\text { Dong (2007) }\end{array}$ \\
\hline $\begin{array}{l}\text { Busca por manter a vantagem competitiva; utilizar o m-commerce } \\
\text { como forma de integrar os processos empresariais para melhorar o } \\
\text { desempenho; e proporcionar informação aos clientes. }\end{array}$ & $\begin{array}{c}\text { Revisão de } \\
\text { literatura com } \\
\text { estudo de } \\
\text { caso }\end{array}$ & $\begin{array}{c}\text { Shih e } \\
\text { Huang } \\
\text { (2009) }\end{array}$ \\
\hline $\begin{array}{l}\text { Ambiente político e legal; políticas organizacionais; infraestrutura de } \\
\text { TIC; cultura organizacional; vantagens relativas; usabilidade } \\
\text { percebida; facilidade de uso percebida; complexidade; } \\
\text { compatibilidade; capacidade da inovação de melhorar o } \\
\text { desempenho individual; suporte da cúpula gerencial; segurança das } \\
\text { informações e propriedade intelectual; confiança; fatores sociais; e } \\
\text { normas subjetivas. }\end{array}$ & $\begin{array}{c}\text { Revisão de } \\
\text { literatura }\end{array}$ & $\begin{array}{c}\text { Houghton e } \\
\text { Sanzogni } \\
\text { (2012) }\end{array}$ \\
\hline
\end{tabular}

Fonte: Elaborado pelos autores (2015).

Em relação aos benefícios e efeitos obtidos com a oferta do serviço de $m$-commerce (segundo grupo), é o Quadro 4 que resume os principais resultados reportados nos artigos.

Quadro 4-Benefícios / Efeitos obtidos com oferta do serviço de m-commerce

\begin{tabular}{|c|c|c|}
\hline $\begin{array}{l}\text { Benefícios / Efeitos obtidos com a oferta do serviço de } m \text { - } \\
\text { commerce }\end{array}$ & $\begin{array}{l}\text { Tipo de } \\
\text { estudo }\end{array}$ & Referência \\
\hline $\begin{array}{l}\text { Benefícios: Utilizar da localização do usuário para comunicar-lhe } \\
\text { um preço específico (oferta exclusiva a um usuário em particular } \\
\text { comunicando-lhe o preço por meio do dispositivo móvel); pode-se } \\
\text { considerar o local e o tempo em que os consumidores se } \\
\text { encontram. } \\
\text { Efeitos: Precificação dos produtos no varejo e competição tendem } \\
\text { a ser mais complexos; numa perspectiva de marketing, o m- } \\
\text { commerce promete maior liberdade de precificação quando se } \\
\text { formula uma estratégia de preço no varejo. }\end{array}$ & Teórico & $\begin{array}{c}\text { Balasubramania } \\
\text { n, Peterson e } \\
\text { Jarvenpaa } \\
\text { (2002) }\end{array}$ \\
\hline $\begin{array}{l}\text { Benefícios: Criação de uma zona de comércio sem fio (shopping } \\
\text { virtual); "deslocamento" da loja para onde seu contato com os } \\
\text { clientes for maior; personalização - baseada na localização do } \\
\text { usuário e no nível psicológico do indivíduo (monitoramento das } \\
\text { atividades do usuário gera dados que aprimoram os filtros, } \\
\text { permitindo oferecer-lhe produtos e serviços nos quais ele } \\
\text { realmente possa estar interessado); utilidade promocional - } \\
\text { divulgar promoções com base nas zonas criadas e na } \\
\text { personalização; nível de interação entre vendedores e }\end{array}$ & Teórico & $\begin{array}{l}\text { Jukic et al. } \\
\quad(2002)\end{array}$ \\
\hline
\end{tabular}




\begin{tabular}{|c|c|c|}
\hline $\begin{array}{l}\text { Benefícios / Efeitos obtidos com a oferta do serviço de } m \text { - } \\
\text { commerce }\end{array}$ & $\begin{array}{l}\text { Tipo de } \\
\text { estudo }\end{array}$ & Referência \\
\hline \multicolumn{3}{|l|}{$\begin{array}{l}\text { compradores - o m-commerce pode agilizar a interação entre } \\
\text { vendedores e compradores, preencher as lacunas deixadas pelo e- } \\
\text { commerce, além de diminuir o tempo entre a propaganda e a } \\
\text { operação de venda. } \\
\text { Efeitos: Fornecedores podem ser forçados a estenderem suas } \\
\text { operações para regiões maiores para aumentar a interação com os } \\
\text { clientes - essa expansão deve ser direcionada no sentido de estar } \\
\text { presente em mais de uma zona, não no sentido de ter uma forte } \\
\text { presença em apenas uma zona em particular. }\end{array}$} \\
\hline $\begin{array}{l}\text { Benefícios: Usuário sempre conectado; possibilidade de } \\
\text { reconhecimento da localização do usuário; serviços personalizados } \\
\text { (baseados na localização e no comportamento do usuário); } \\
\text { geração de valor para a empresa; e serviços com valor adicionado } \\
\text { (por meio da criação de novos modelos de negócio ou modificação } \\
\text { dos modelos existentes). }\end{array}$ & Teórico & $\begin{array}{l}\text { Yeo e Huang } \\
\text { (2003) }\end{array}$ \\
\hline Benefícios: Demonstrar a capacidade de inovação. & $\begin{array}{l}\text { Revisão } \\
\text { de } \\
\text { literatura }\end{array}$ & $\begin{array}{l}\text { Schierholz, } \\
\text { Kolbe e Brenner } \\
\text { (2005) }\end{array}$ \\
\hline $\begin{array}{l}\text { Benefícios: Localização; personalização; omnipresença; e } \\
\text { pontualidade. }\end{array}$ & $\begin{array}{l}\text { Revisão } \\
\text { de } \\
\text { literatura } \\
\text { com } \\
\text { estudo de } \\
\text { caso }\end{array}$ & $\begin{array}{l}\text { Huang, Qi e } \\
\text { Dong (2007) }\end{array}$ \\
\hline $\begin{array}{l}\text { Benefícios: Marketing direcionado com base na localização e } \\
\text { características do usuário. }\end{array}$ & Teórico & Clarke (2008) \\
\hline \multirow{2}{*}{$\begin{array}{l}\text { Benefícios: Personalização de serviços baseada na localização do } \\
\text { usuário (location-based) e no contexto (context-awareness). }\end{array}$} & $\begin{array}{l}\text { Estudo de } \\
\text { caso com } \\
\text { proposta }\end{array}$ & $\begin{array}{l}\text { Georgiadis } \\
\text { (2009) }\end{array}$ \\
\hline & $\begin{array}{l}\text { Estudo de } \\
\text { caso }\end{array}$ & $\begin{array}{c}\text { Sharma e } \\
\text { Gutiérrez (2010) }\end{array}$ \\
\hline $\begin{array}{l}\text { Benefícios: Envio de mensagens personalizadas (marketing) aos } \\
\text { usuários com base nos hábitos de navegação e nos padrões de } \\
\text { compra. }\end{array}$ & $\begin{array}{l}\text { Estudo de } \\
\text { caso com } \\
\text { proposta }\end{array}$ & Lee e Ho (2010) \\
\hline $\begin{array}{l}\text { Benefícios: Marketing baseado na localização (location-based } \\
\text { marketing) está exercendo grande papel no m-commerce } \\
\text { relacionado às táticas de marketing dos principais varejistas; } \\
\text { Inovação em marketing; redução de custos; e oportunidade de } \\
\text { atingir melhores consumidores. }\end{array}$ & Survey & $\begin{array}{l}\text { Tomášková } \\
\text { (2010) }\end{array}$ \\
\hline $\begin{array}{l}\text { Benefícios: Geração de dados em massa [pelo m-commerce] } \\
\text { combinado com Business Intelligence (BI) gera: informação } \\
\text { confiável; operação personalizada; informação instantânea sobre o }\end{array}$ & $\begin{array}{l}\text { Teórico } \\
\text { com }\end{array}$ & $\begin{array}{l}\text { Gan, Tu e Wang } \\
\text { (2013) }\end{array}$ \\
\hline
\end{tabular}




\begin{tabular}{|c|c|c|}
\hline $\begin{array}{l}\text { Benefícios / Efeitos obtidos com a oferta do serviço de } m \text { - } \\
\text { commerce }\end{array}$ & $\begin{array}{l}\text { Tipo de } \\
\text { estudo }\end{array}$ & Referência \\
\hline $\begin{array}{l}\text { desempenho; descrição e classificação do comportamento dos } \\
\text { consumidores; geração de marketing personalizado (a partir do BI); } \\
\text { e oferta de informação e serviços customizados para melhorar a } \\
\text { experiência do usuário. }\end{array}$ & proposta & \\
\hline
\end{tabular}

Fonte: Elaborado pelos autores (2015).

Em relação ao terceiro grupo, que apresenta as barreiras para a oferta do serviço de $m$ commerce, o Quadro 5 apresenta os principais resultados reportados nos artigos.

Quadro 5 - Barreiras para a oferta do serviço de m-commerce

\begin{tabular}{|c|c|c|}
\hline Barreiras para a oferta do serviço de $m$-commerce & $\begin{array}{l}\text { Tipo de } \\
\text { estudo }\end{array}$ & Referência \\
\hline $\begin{array}{l}\text { Diferentes padrões de tecnologia; segurança; e baixa velocidade de } \\
\text { conexão. }\end{array}$ & Teórico & $\begin{array}{l}\text { Yeo e } \\
\text { Huang } \\
\text { (2003) }\end{array}$ \\
\hline $\begin{array}{l}\text { Diferentes padrões de tecnologia; diferentes sistemas operacionais; } \\
\text { segurança; baixa velocidade de conexão; interfaces gráficas não } \\
\text { adaptadas para telas pequenas; custo da conexão sem fio; estratégias } \\
\text { de marketing inadequadas; nível de conforto do usuário; questões } \\
\text { culturais e comportamento do consumidor. }\end{array}$ & $\begin{array}{l}\text { Revisão } \\
\text { de } \\
\text { literatura } \\
\text { com } \\
\text { estudo de } \\
\text { caso }\end{array}$ & $\begin{array}{c}\text { Huang, Qi } \\
\text { e Dong } \\
\text { (2007) }\end{array}$ \\
\hline $\begin{array}{l}\text { Rivalidade entre regimes de conhecimento (regime tecnológico e regime } \\
\text { orientado para os negócios); complexidade tecnológica subestimada; } \\
\text { incerteza sobre as necessidades do mercado e dos clientes (também } \\
\text { em relação à qualidade do serviço); modelo de negócios inadequado; } \\
\text { falta de convergência entre instituições - o desenvolvimento do m- } \\
\text { commerce requer a convergência de várias instituições e mecanismos } \\
\text { que atualmente encontram-se separados a autônomos, logo, políticas e } \\
\text { estratégias precisam ser desenvolvidas para superar isso. }\end{array}$ & $\begin{array}{l}\text { Estudo de } \\
\text { caso }\end{array}$ & $\begin{array}{c}\text { Godoe e } \\
\text { Hansen } \\
(2009)\end{array}$ \\
\hline $\begin{array}{l}\text { Lei da privacidade impede serviços baseados na localização (no caso } \\
\text { do país estudado). }\end{array}$ & $\begin{array}{l}\text { Estudo de } \\
\text { caso com } \\
\text { proposta }\end{array}$ & $\begin{array}{l}\text { Lee e Ho } \\
(2010)\end{array}$ \\
\hline $\begin{array}{l}\text { Modelos de negócio inadequado (modelos orientados para o e- } \\
\text { commerce não são necessariamente modelos para o } m \text {-commerce, pois } \\
\text { a cadeia de valor para o m-commerce é muito mais complexa); } \\
\text { diferentemente do e-commerce, no m-commerce o usuário não está } \\
\text { livre para acessar Internet, ele depende de empresas de } \\
\text { telecomunicação móvel e de serviços de dados, além disso, o usuário é } \\
\text { cobrado pelo volume de dados que trafegam em sua conexão; custo do } \\
\text { serviço de } m \text {-commerce é ditado pelo provedor de comunicação móvel. }\end{array}$ & $\begin{array}{l}\text { Estudo de } \\
\text { caso }\end{array}$ & $\begin{array}{c}\text { Sharma e } \\
\text { Gutiérrez } \\
(2010)\end{array}$ \\
\hline
\end{tabular}

Fonte: Elaborado pelos autores (2015). 
Por fim, em relação ao quarto grupo - fatores críticos de sucesso na oferta do serviço de $m$ commerce-, o Quadro 6 apresenta um resumo dos resultados reportados nos artigos.

Quadro 6 - Fatores críticos de sucesso na oferta do serviço de m-commerce

\begin{tabular}{|c|c|c|}
\hline Fatores Críticos de Sucesso & $\begin{array}{l}\text { Tipo de } \\
\text { estudo }\end{array}$ & Referência \\
\hline $\begin{array}{l}\text { A adoção da tecnologia deve considerar se realmente há mercado } \\
\text { suficiente para justificar a adoção da tecnologia em questão; deve } \\
\text { haver um equilíbrio entre possibilidades técnicas e otimização } \\
\text { econômica. }\end{array}$ & Teórico & $\begin{array}{l}\text { Balasubramania } \\
\text { n, Peterson e } \\
\text { Jarvenpaa } \\
\text { (2002) }\end{array}$ \\
\hline $\begin{array}{l}\text { M-commerce deve ser parte da estratégia corporativa e estar } \\
\text { embutido na estratégia de marketing; utilização de componentes } \\
\text { existentes e implementação baseada em produtos-padrão para } \\
\text { manter os custos baixos; cadeia de valor exerce um papel importante } \\
\text { (desde que a terceirização seja um facilitador para economia de } \\
\text { tempo e custo); simplicidade; e desempenho. }\end{array}$ & $\begin{array}{l}\text { Estudo } \\
\text { de caso }\end{array}$ & $\begin{array}{l}\text { Reichold et al. } \\
\qquad(2003)\end{array}$ \\
\hline $\begin{array}{l}\text { Iniciar com uma estratégia de necessidade, não com uma estratégia } \\
\text { tecnológica; desenvolver uma estratégia eletrônica que complemente } \\
\text { a estratégia empresarial; entender e focar os clientes certos; utilizar } \\
\text { os recursos sabiamente; garantir segurança e credibilidade aos } \\
\text { clientes; proporcionar uma experiência completa aos clientes; } \\
\text { entregar serviços personalizados; proporcionar conveniência aos } \\
\text { clientes; e ter um modelo de negócio adequado. }\end{array}$ & $\begin{array}{l}\text { Revisão } \\
\text { de } \\
\text { literatur } \\
\text { a com } \\
\text { estudo } \\
\text { de caso }\end{array}$ & $\begin{array}{l}\text { Huang, Qi e } \\
\text { Dong (2007) }\end{array}$ \\
\hline $\begin{array}{l}\text { Tecnológico - Estratégia do 'produto' móvel; qualidade da } \\
\text { tecnologia de informação; hardware; software; e capacidade da } \\
\text { conexão sem fio. } \\
\text { Organizacional - Vantagem competitiva; apoio à gestão; cultura e } \\
\text { ambiente. } \\
\text { Social - Influência social; Gestão do relacionamento com o } \\
\text { consumidor (CRM). } \\
\text { Pessoal - Comportamento e auto-eficácia; estratégias de tarefa; e } \\
\text { consideração de restrições pessoais. }\end{array}$ & $\begin{array}{l}\text { Estudo } \\
\text { de caso }\end{array}$ & $\begin{array}{c}\text { Shih e Huang } \\
\text { (2009) }\end{array}$ \\
\hline $\begin{array}{l}\text { Relacionados ao serviço: Proporcionar a melhor experiência ao } \\
\text { usuário em questões de facilidade de uso, acessibilidade, serviços } \\
\text { oferecidos (integralidade das atividades de cadeia de valor), } \\
\text { proposição de valor aos clientes; opções de pagamento facilitadas; } \\
\text { habilidade do modelo de negócios mudar e evoluir com o tempo - } \\
\text { dinamicidade; empresa deve ajustar-se a diferentes parâmetros de } \\
\text { mercado; modelo de negócio deve ser centrado no usuário } \\
\text { (colocando o potencial cliente em primeiro lugar); interface boa, fácil } \\
\text { de usar e acessível pode ser um fator decisivo para o sucesso do } \\
\text { modelo de negócios, pois quanto mais utilizável é a interface, mais } \\
\text { viável torna-se o modelo de negócios e maior é a usabilidade } \\
\text { percebida (uma boa interface pode elevar significativamente a } \\
\text { proposição de valor). }\end{array}$ & $\begin{array}{l}\text { Estudo } \\
\text { de caso }\end{array}$ & $\begin{array}{c}\text { Sharma e } \\
\text { Gutiérrez (2010) }\end{array}$ \\
\hline
\end{tabular}




\begin{tabular}{|l|c|c|}
\hline \multicolumn{1}{|c|}{ Fatores Críticos de Sucesso } & $\begin{array}{l}\text { Tipo de } \\
\text { estudo }\end{array}$ & Referência \\
\hline $\begin{array}{l}\text { Relacionados à organização: Interesse em colaborar e formar } \\
\text { parcerias com outros atores da indústria; estrutura de custos } \\
\text { adequada e padronizada, juntamente a um modelo de organização; } \\
\text { bom e suficiente retorno sobre o investimento para cada parceiro de } \\
\text { negócio; colaboração e parcerias; e capacidade de resposta às } \\
\text { tendências de mercado. }\end{array}$ & \begin{tabular}{l} 
\\
\hline $\begin{array}{l}\text { Conhecer a carteira de clientes; fazer uma segmentação adequada } \\
\text { para personalizar o conteúdo; adaptar as estratégias para uso de } \\
\text { dispositivos móveis para comércio, com a receptividade dos clientes; } \\
\text { considerar a importância da análise de viabilidade do m-commerce } \\
\text { para cada setor e cada caso em específico; considerar o ajuste } \\
\text { tecnológico requerido; analisar a receptividade do m-marketing e } \\
\text { compras à distância pela carteira de clientes da empresa. }\end{array}$
\end{tabular} & $\begin{array}{c}\text { Survey } \\
\text { Catalán e } \\
\text { Ramón- }\end{array}$ \\
\hline
\end{tabular}

Fonte: Elaborado pelos autores (2015).

Apresentados os principais resultados reportados nos artigos analisados, no tópico seguinte apresenta-se uma interpretação desses dados à luz da literatura de inovação apresentada no referencial teórico deste trabalho.

\section{ANÁLISE}

Retomando as classificações sobre os graus de inovação (OCDE, 2005; CHRISTENSEN, 2012), considera-se que o m-commerce é uma inovação de ruptura, pois várias de suas características são alinhadas ao que Christensen (2012) aponta como características das inovações de ruptura. O primeiro ponto é que uma inovação de ruptura leva a pior desempenho dos produtos (CHRISTENSEN, 2012), o que fica evidente quando Yeo e Huang (2003) e Huang, Qi e Dong (2007) falam dos diferentes padrões de tecnologia, problemas com segurança da informação, baixa velocidade da conexão, interfaces não adaptadas para telas pequenas, custo da conexão e, quando Sharma e Gutiérrez (2010) afirmam que os clientes não estão livres para acessar a Internet, pois dependem dos operadores de telecomunicações. Entretanto, mesmo com pior desempenho e outras dificuldades, esses produtos/serviços têm aceitação do mercado, pois apresentam outras características, que trazem proposições de valor bastante diferentes daquelas que existiam anteriormente, proporcionando valor aos clientes (CHRISTENSEN, 2012). Umas dessas características é a possibilidade do indivíduo permanecer conectado em qualquer lugar (YEO; HUANG, 2003). Aqui não são apresentadas mais características, pois o presente estudo não tem objetivo de focar no usuário final, embora fosse importante para trazer mais detalhes a serem confrontados com a literatura existente.

Revisando os cinco princípios da inovação de ruptura apontados por Christensen (2012), observa-se que o m-commerce também abrange alguns deles: as companhias dependem dos clientes - Balasubramanian, Peterson e Jarvenpaa (2002) e Huang, Qi e Dong (2007) argumentam numa linha de que deve haver mercado suficiente para justificar os investimentos e a adoção da 
tecnologia; mercados que não existem não podem ser avaliados - Godoe e Hansen (2009) discorrem sobre as incertezas das necessidades do mercado; e as capacidades de uma organização definem suas incapacidades - Sharma e Gutiérrez (2010) argumentam que modelos de negócio orientados para o comércio eletrônico tradicional não são necessariamente adequados para o $m$-commerce, visto que a cadeia de valor para este último é muito mais complexa. Com base nas peculiaridades que os autores citados trazem, observa-se que o m-commerce não poderia ser considerado uma inovação incremental ou radical, pois suas caraterísticas são mais próximas do que Christensen (2012) denomina inovação de ruptura.

Partindo para o foco das inovações (SUNDBO; GALLOUJ, 1998; OCDE, 2005), verifica-se que o $\mathrm{m}$-commerce não está focado em apenas uma dimensão. Conforme apontam os resultados dos artigos analisados, o m-commerce pode abranger: uma inovação de produto ou serviço - a empresa passa a oferecer um novo tipo de serviço, que, mesmo sendo um serviço em massa, pode ser personalizado (BALASUBRAMANIAN; PETERSON; JARVENPAA, 2002; JUKIC et al., 2002; YEO; HUANG, 2003; HUANG; QI; DONG 2007; CLARKE, 2008; GEORGIADIS, 2009; LEE; HO, 2010; SHARMA; GUTIÉRREZ, 2010; TOMÁŠKOVÁ, 2010; GAN; TU; WANG, 2013); uma inovação de processo - Shih e Huang (2009) falam sobre integração de processos por meio do $\mathrm{m}$-commerce e a utilização disso como meio de fornecer informação aos clientes, e, quando Martín, López-Catalán e Ramón-Jerónimo (2012) falam sobre necessidade de ajuste tecnológico, compras à distância, competência tecnológica e adaptação das atividades da empresa ao m-commerce, percebe-se que por trás dessas variáveis existe a ideia de inovação em processo; uma inovação organizacional - a literatura consultada não apresenta o m-commerce em si como uma inovação organizacional, entretanto, Godoe e Hansen (2009) e Sharma e Gutiérrez (2010) afirmam que é necessário ter um modelo de negócios que atenda às necessidades do serviço em questão, dessa forma, em alguns casos, pode ocorrer uma inovação organizacional ocasionada pela intenção de oferta do serviço de m-commerce, além disso, Yeo e Huang (2003) também falam da criação de um novo modelo de negócios para oferecer serviços com valor adicionado; uma inovação de mercado ou de marketing o m-commerce permite a realização de marketing personalizado, considerando localização, características e/ou contexto em que os usuários de dispositivos móveis encontram-se (BALASUBRAMANIAN; PETERSON; JARVENPAA, 2002; JUKIC et al., 2002; YEO; HUANG, 2003; HUANG; QI; DONG, 2007; CLARKE, 2008; GEORGIADIS, 2009; LEE; HO, 2010; SHARMA; GUTIÉRREZ, 2010; TOMÁŠKOVÁ, 2010; GAN; TU; WANG, 2013); e uma inovação ad hoc - Martín, López-Catalán e Ramón-Jerónimo (2012) afirmam que, para a oferta do serviço de m-commerce pelas empresas, cada setor e cada caso precisam ser avaliados em específico, o que indica que a implantação do serviço de $m$-commerce como inovação empresarial é uma inovação personalizada, no entanto, Sundbo e Gallouj (1998) propõe que uma inovação ad hoc é uma inovação desenvolvida em parceria entre cliente e fornecedor e, como na implantação do serviço de m-commerce não necessariamente existe um fornecedor, considera-se que sua implantação somente pode ser considerada uma inovação ad hoc se ela for conduzida com o auxílio de um fornecedor de serviços de consultoria ou de apoio. 
Com isso, verifica-se que a implementação do serviço de $m$-commerce nas empresas é uma tarefa complexa, pois ela não requer mudanças em apenas uma dimensão. $O$ sucesso na implementação da tecnologia em questão, conforme os trabalhos analisados, requer mudanças na forma da empresa criar, fazer e conduzir seus negócios. Considerando a literatura de inovação, podese dizer que o m-commerce é uma inovação em diversos aspectos, tanto em negócio, como em marketing, processo e gestão. Em outras palavras, a implementação do serviço de $m$-commerce é uma inovação que pode ser vista sob vários pontos de vista.

Partindo para a análise da questão estratégica, Reichold et al. (2003) e Huang, Qi e Dong (2007) afirmam que a estratégia eletrônica desenvolvida pela empresa deve ser parte da estratégia empresarial e não uma estratégia a parte, que é a mesma ideia adotada na literatura sobre inovação (DAVILA; EPSTEIN; SHELTON, 2007; HIDALGO; ALBORS, 2008). Assim, mesmo que os autores das pesquisas sobre $m$-commerce não recorram à literatura de inovação empresarial, percebe-se que muitas das variáveis que eles trazem são compatíveis às que os autores de inovação apresentam.

Já quanto ao tipo de atividades inovativas (DAVILA; EPSTEIN; SHELTON, 2007), o mcommerce compreende a atividade tecnológica - desenvolvimento de novos serviços (JUKIC et al., 2002; YEO; HUANG, 2003; HUANG; QI; DONG, 2007; CLARKE, 2008; LEE; HO, 2010; GAN; TU; WANG, 2013) - e a atividade estratégica, que é a definição do modelo de negócios. Este último pode orientar a inovação em relação à proposição de valor aos clientes - que é discutida no trabalho de Yeo e Huang (2003) - e cliente-alvo - que aparece no trabalho de Jukic et al. (2002), quando comentam da entrada em novos mercados utilizando o m-commerce, e no trabalho de Tomášková (2010), quando o autor afirma que o m-commerce abre oportunidades de atingir melhores clientes.

As barreiras à inovação (HIDALGO; ALBORS, 2008; TIDD; BESSANT; PAVITT, 2008) também aparecem nas pesquisas de m-commerce. Lee e Ho (2010) apontam para a existência de uma barreira bastante específica, relacionada à legislação, que não impede o m-commerce por completo, mas impede algumas de suas funcionalidades. Barreiras mais gerais são apontadas por Huang, Qi e Dong (2007), Godoe e Hansen (2009) e Sharma e Gutiérrez (2010). Entre os argumentos desses autores, aparecem questões como modelos de negócio inadequado, complexidade da cadeia de valor, questões culturais e de comportamento do consumidor, custo, rivalidade entre regimes de conhecimento tecnológico e regime de conhecimento orientado para os negócios, complexidade tecnológica subestimada e incerteza sobre as necessidades dos clientes. Essas barreiras já foram discutidas pelos pesquisadores de inovação citados nesta pesquisa, o que indica que não são dificuldades enfrentadas apenas pelo m-commerce, mas dificuldades relacionadas a qualquer processo de inovação.

Para superar algumas dessas barreiras, Davila, Epstein e Shelton (2007) e Tidd, Bessant e Pavitt (2008) apontam para a importância das colaborações, o que também foi identificado nas pesquisas sobre m-commerce, mais especificamente nos trabalhos de Godoe e Hansen (2009) e Sharma e Gutiérrez (2010). Entre os argumentos desses autores, está que a colaboração e parcerias são fundamentais, pois o mercado móvel é muito mais um mercado dominado por empresas de telecomunicação do que um mercado aberto. 
Além disso, verifica-se que as duas dimensões que levam as empresas a inovar (TIDD; BESSANT; PAVITT, 2008), também aparecem nas pesquisas sobre $m$-commerce. Na dimensão tecnológica, pode-se considerar a importância competitiva e a complexidade da inovação. No que diz respeito à importância competitiva, Huang, Qi e Dong (2007) e Shih e Huang (2009) discorrem sobre vantagem competitiva por meio da melhoria do relacionamento com os clientes, integração dos processos e aumento nas receitas da empresa. E em relação à complexidade da inovação, Martín, López-Catalán e Ramón-Jerónimo (2012) chamam a atenção para a competência tecnológica como fator crítico de sucesso, Godoe e Hansen (2009) e Sharma e Gutiérrez (2010) discorrem sobre a importância de formar parcerias e, no estudo de Reichold et al. (2003), constatou-se que a utilização de componentes existentes e implementação baseada em produtos-padrão foram fundamentais para manter os custos baixos e ter sucesso na implementação do serviço de $m$-commerce. Esses argumentos apontam todos na mesma direção - a complexidade na implantação do serviço.

Já na dimensão de herança da empresa, é citada a estratégia corporativa, as capacidades e conhecimento técnico e a cultura da empresa. A estratégia corporativa aparece em vários trabalhos, focando principalmente na necessidade da estratégia eletrônica ser parte da estratégia corporativa (REICHOLD et al., 2003; HUANG; QI; DONG, 2007) e, numa linha diferente, Schierholz, Kolbe e Brenner (2005) afirmam que a adoção de uma nova tecnologia pode demonstrar a capacidade de inovação de uma empresa, o que também pode ser tomado como uma estratégia empresarial. $\mathrm{O}$ segundo tópico, capacidades e conhecimento técnico, aparece no trabalho de Martín, López-Catalán e Ramón-Jerónimo (2012), quando falam da competência tecnológica requerida, no trabalho de Shih e Huang (2009), quando expõem a necessidade da organização conhecer a tecnologia da informação, e no trabalho de Sharma e Gutiérrez (2010), quando discutem a importância da adequação da interface gráfica do software. Esses dois últimos argumentos reportam a capacidade técnica necessária e, quanto mais as empresas possuírem essas capacidades, maior será a probabilidade de terem sucesso com o serviço de m-commerce, visto que são apontados como fatores críticos de sucesso. Por último, a cultura da empresa é lembrada no trabalho de Shih e Huang (2009), quando falam do apoio à gestão, cultura e ambiente, e no trabalho de Alfahl, Houghton e Sanzogni (2012), quando apontam que a cultura da empresa é um fator que pode influenciar a oferta do m-commerce.

Com base no que foi apresentado neste tópico, verifica-se que grande parte da literatura sobre inovação empresarial aplica-se também ao serviço de m-commerce. O próximo tópico é reservado às considerações finais desta pesquisa.

\section{CONSIDERAÇÕES FINAIS}

Conforme visto, este artigo trouxe à discussão um campo de pesquisa no $m$-commerce que carece de desenvolvimento e que está relacionado à utilização da literatura de inovação nas pesquisas sobre o serviço móvel. Como visto no tópico introdutório, foi objetivo deste artigo analisar se a literatura de inovação é aplicável aos estudos sobre $m$-commerce que analisam os ofertantes do serviço. 
Revisando as pesquisas que analisam os ofertantes do serviço de $m$-commerce, verificou-se que a literatura de inovação é aplicável ao caso do serviço móvel. Os resultados apontam para a existência de fatores convergentes entre a literatura de inovação empresarial e a literatura de $\mathrm{m}$ commerce. Identificou-se que as classificações estabelecidas naquela literatura são aplicáveis ao $\mathrm{m}$ commerce, permitindo seu posicionamento dentro das mesmas.

$\mathrm{O}$ fato de os artigos sobre $m$-commerce não recorrerem à literatura de inovação empresarial aponta para uma lacuna de conhecimento nas pesquisas sobre o serviço móvel. Utilizar-se dos conhecimentos que a literatura de inovação construiu ao decorrer de sua história seria frutífero ao corpo de conhecimentos sobre o m-commerce, como é possível concluir a partir desta pesquisa.

Conforme visto, as pesquisas sobre m-commerce analisadas neste estudo têm gerado conhecimentos já anteriormente encontrados na literatura de inovação empresarial. Utilizar-se de uma literatura mais desenvolvida (neste caso, a de inovação empresarial), possivelmente geraria um avanço na literatura de m-commerce e, tendo uma base de conhecimento comum já definida, permitiria que as pesquisas sobre $m$-commerce se voltassem às particularidades do serviço e não às generalidades já conhecidas. Isso contribuiria para estabelecer e solidificar esse novo corpo de conhecimentos. A partir deste ponto de vista, muito do que os autores dos trabalhos analisados têm feito pode ser considerado retrabalho. Partir da literatura geral de inovação empresarial e direcionar as pesquisas para o m-commerce traria contribuições teóricas importantes para o campo.

Dessa forma, este trabalho buscou contribuir para o preenchimento desta lacuna do conhecimento e espera-se ter dado uma indicação de pesquisa aos pesquisadores de ambos os campos (m-commerce e inovação empresarial). Além de contribuir com o crescimento do conhecimento acadêmico, os resultados atingidos por esta pesquisa também mostram utilidade prática, a qual consiste na utilização dos conhecimentos de inovação empresarial para uma implementação de sucesso dos serviços de m-commerce.

Pesquisas que analisam os ofertantes do serviço de $m$-commerce, conforme visto, não têm sido muito numerosas, mas isso não reduz a importância do tema, pois, como visto na introdução, especificamente no Brasil, a taxa de crescimento desse mercado tem ultrapassado as duas casas decimais. Por esse motivo, deixa-se como recomendações para pesquisas futuras a realização de estudos que aprofundem o conhecimento sobre os ofertantes do serviço de $m$-commerce, baseandose na literatura de inovação empresarial já disponibilizada. Tal aprofundamento pode ser realizado por meio de estudos de casos únicos ou múltiplos, analisando organizações que passaram a oferecer o serviço de $m$-commerce e focando nas particularidades desse serviço. Estudos quantitativos sobre os ofertantes do serviço de $m$-commerce podem ser realizados com base na literatura de inovação empresarial e nas particularidades do serviço apresentadas neste artigo, entretanto, entende-se que, por enquanto, ainda é necessário um aprofundamento maior na compreensão acerca do tema. Outra recomendação de pesquisa é analisar o m-commerce à luz da literatura de marketing sobre relacionamento com o consumidor, visto que no serviço de $m$-commerce existe a possibilidade de interação entre empresa e consumidor. 


\section{REFERÊNCIAS}

ALFAHL, H.; HOUGHTON, L.; SANZOGNI, L. Mobile Commerce adoption in organizations: a literature review e future research directions. Journal of Electronic Commerce in Organizations, v. 10, p. 61+, 2012.

BALASUBRAMANIAN, S.; PETERSON, R. A.; JARVENPAA, S. L. Exploring the implications of mcommerce for markets e marketing. Journal of the Academy of Marketing Science, v. 30, n. 4, p. 348361, 2002.

BHEDA, N. M-commerce for the masses. Siliconindia, n. 40, July, 2010.

CHANG, M. K.; CHEUNG, W.; LAI, V. S. Literature derived reference models for the adoption of online shopping. Information \& Management, v. 42, p. 543-559, 2005.

CHONG, A. Y.-L. A two-staged SEM-neural network approach for understanding e predicting the determinants of m-commerce adoption. Expert Systems with Applications, v. 40, p. 1240-1247, 2013.

CHRISTENSEN, C. M. O dilema da inovação: quando as novas tecnologias levam empresas ao fracasso. São Paulo: M. Books do Brasil Editora, 2012. Tradução: Laura Prates Veiga.

CLARKE, I., III. Emerging value propositions for m-commerce. Journal of Business Strategies, v. 25, n. 2 , p. $41+, 2008$.

DAVILA, T.; EPSTEIN, M. J.; SHELTON, R. As regras da inovação. Porto Alegre: Bookman, 2007. Tradução: Raul Rubenich.

E-BIT. Relatório WebShoppers. 31. ed., 2015. Disponível em: <http://www.webvenda.com/wpcontent/uploads/2015/02/31_webshoppers.pdf>.

FROLICK, M. N.; CHEN, L. Assessing m-commerce opportunities. Information systems management. Spring, 2004.

GAN, L.; TU, W.; WANG, A. The Research on Application of Business Intelligence in Mobile Ecommerce. Proceedings of the 2013 International Academic Workshop on Social Science (law-Sc 2013), v. 50, p. 331-334, 2013.

GEORGIADIS, C. K. Mobile Commerce Application Development: Implementing Location-aware Information Services. In: GERACI, P.; LOGOTHETIS, M. Aict: 2009 Fifth Advanced International Conference on Telecommunications, p. 333-338, 2009.

GODOE, H.; HANSEN, T. B. Technological regimes in m-commerce: Convergence as a barrier to diffusion e entrepreneurship? Telecommunications Policy, v. 33, n. 1/2, p. 19-28, 2009.

HIDALGO, A.; ALBORS, J. Innovation management techniques and tools: a review from theory and practice. R\&D Management, v. 38, n. 2, p. 113-127, 2008.

HUANG, W.; QI, L. Y.; DONG, L. H. Business Models e Implementations of M-Commerce: Case Studies e Future Research Issues. 2007 International Conference on Wireless Communications, Networking and Mobile Computing, p. 3637-3640, 2007.

IVAN, I.; MILODIN, D.; ZAMFIROIU, A. Security of m-commerce transactions. Theoretical and Applied Economics, v. 20, n. 7, p. 59-76, 2013.

JIN, C. H.; VILLEGAS, J. Mobile phone user's behaviors: the motivation factors of the mobile phone user. International Journal of Mobile Marketing, v. 3, n. 2, p. 4-14, 2008.

JONKER, J.-W. M-commerce e m-payment: combining technologies. Bedrijfswiskunde en informatica, p. 1-28, 2003.

Estudos do CEPE [ISSN 1982-6729]. Santa Cruz do Sul, n. 44, p. 127-146, jul./dez. 2016. https://online.unisc.br/seer/index.php/cepe/index 
JUKIC, N. et al. M-commerce: Analysis of impact on marketing orientation. In: KHOSROWPOUR, M. Issues and Trends of Information Technology Management in Contemporary Organizations, p. 305307, 2002.

LEE, C.-S.; HO, J. C. A Framework for Analyzing Business Model Innovation in Mobile Commerce. Journal of International Technology \& Information Management, v. 19, n. 4, p. 37-60, 2010.

MALIK, A; KUMRA, R.; SRIVASTAVA, V. Determinants of consumer acceptance of m-commerce. South Asian Journal of Management, v. 20, n. 2, p. 102-126, 2013.

MARTíN, S. S.; LÓPEZ-CATALÁN, B.; RAMÓN-JERÓNIMO, M. A. Factors determining firms' perceived performance of mobile commerce. Industrial Management \& Data Systems, v. 112, n. 6, p. 946-963, 2012.

MARTÍN-GUTIÉRREZ, S.; LÓPEZ-CATALÁN, S.; RAMÓN-JERÓNIMO, M. A. Determinants of involvement in mobile commerce: the moderating role of gender. EsicMarket, v. 141, p. 69-101, 2012.

MAY, P. Mobile Commerce: Opportunities, Applications, e Technologies of Wireless Business. Breakthroughs in application development series. New York: Cambridge University Press, 2001. 304p.

NGAI, E.W.T.; GUNASEKARAN, A. E-commerce in Hong Kong: an empirical perspective e analysis. Internet Research, v.15, n. 2, p. 141-159, 2005.

NGAI, E. W. T.; GUNASEKARAN, A. A review for mobile commerce research e applications. Decision Support Systems, v. 43, n. 1, p. 3-15, 2007.

NYBAKK, E.; JENSSEN, J. I. Innovation strategy, working climate, and financial performance in traditional manufacturing firms: an empirical analysis. International Journal of Innovation Management, v.16, n. 2, 26p., 2012.

OCDE, Organização para a Cooperação e Desenvolvimento Econômico. Manual de Oslo: proposta de diretrizes para coleta e interpretação de dados sobre inovação tecnológica. FINEP, 2005.

REICHOLD, A. et al. Case study - M-commerce at Helsana Health Insurance: Mobile premium calculator. Proceedings of the 14th International Workshop on Database and Expert Systems Applications, p. 877-881, 2003.

SCHIERHOLZ, R.; KOLBE, L.; BRENNER, W. Strategy Alignment of Mobile Solutions in CustomerOriented Processes. Wirtschaftsinformatik, v. 47, n. 1, 2005.

SHARMA, S.; GUTIÉRREZ, J. A. An evaluation framework for viable business models for mcommerce in the information technology sector. Electronic Markets, v. 20, n. 1, p. 33-52, 2010.

SHIH, Y.-Y.; HUANG, S.-S. Exploring the critical success factors of mobile commerce via qualitative method- in case of insurance industry. Proceedings of the 2009 Sixth International Conference on Information Technology: New Generations, n. 1-3, p. 958-962, 2009.

SUNDBO, J.; GALLOUJ, F. Innovation in services. SI4S Project synthesis. Work package 3/4, SI4S, 1998.

TIDD, J.; BESSANT, J.; PAVITT, K. Gestão da Inovação. 3. ed. Porto Alegre: Bookman, 2008. Tradução: Elizamari Rodrigues Becker.

TOMÁŠKOVÁ, H. M-Commerce e M-banking focused on Czech republic. In: MLADENOV, V. et al. (org.). Proceedings of the International Conference on Communication and Management in Technological Innovation and Academic Globalization, p.109-112, 2010. 
VILLAS, M. V.; MACEDO-SOARES, T. D. L. A.; RUSSO, G. M. Bibliographical research method for business administration studies: a model based on scientific journal ranking. Brazilian Administration Review, v. 5, n. 2, p. 139-159, 2008.

WU, J.-H.; WANG, S.-C. What drives mobile commerce? An empirical evaluation of the revised technology acceptance model. Information \& Management, v. 42, p. 719-729, 2005.

YANG, K. C. C. Exploring factors affecting the adoption of mobile commerce in Singapore. Telematics and Informatics, n. 22, p. 257-277, 2005.

YEO, J.; HUANG, W. Mobile E-commerce Outlook. International Journal of Information Technology \& Decision Making, v. 2, n. 2, p. 313-332, 2003.

Estudos do CEPE [ISSN 1982-6729]. Santa Cruz do Sul, n. 44, p. 127-146, jul./dez. 2016. 